somit auch vom physikalischen Standpunkte ein höchst bemerkenswertes Problem vorliegt.

In der Folge achtete ich nun besonders auf diese Erscheinung. Als in verhältnismäßig kurzer Zeit bei der Schädelperkussion in vier weiteren Fällen Schettern gefunden und meine Aufmerksamkeit dadurch auf die Diagnose Hirntumor gelenkt wurde, die dann jedesmal durch Tumorpapille und weiteren Verlauf bestätigt wurde, so glaubte ich das Schettern und die Tympanie des Schädels als pathognomonisch für Hirntumor ansehen zu dürfen. Bei der weiteren systematischen Prüfung der Schädelperkussion bei Kindern fand sich jedoch die Tympanie viel häufiger auch bei anderen Krankheiten (besonders schön bei Meningitis, vor allem hier wieder bei der tuberkulösen Basilarmeningitis), dann auch in ganz verschiedener Stärke, manchmal nur angedeutet, manchmal von einem zum anderen Tage wechselnd, sowohl in der Stärke als im Auftreten überhaupt, soda $B$ ich zeitweilig selbst mit dem Symptom nichts anzufangen wußte. Trotzdem führte ich die Beobachtungen weiter und trug sie fortlaufend in die Krankengeschichten ein.

Als erstes Resultat dieser Beobachtungen wäre hervorzuheben, daß tympanitischer Schall beim Beklopfen luftleerer Organe entstehen kann, doch kann ich heute auf die physikalischen Bedingungen des Phänomens nicht näher eingehen.

Um Selbsttäuschungen zu entgehen, wurde die Perkussion von einem zweiten Beobachter in einiger Entfernung kontrolliert und umgekehrt. Die Perkussion wurde direkt mit dem Finger ohne Plessimeter ausgeführt; um eine Resonanz der Unterlage. auszuschalten, wurde der Kranke im Stehen oder Sitzen, im Liegen nur auf weicher Unterlage untersucht. Die Möglichlseit, da $B$ die Tympanie beim Beklopfen des Schädels durch Resonanz der Mundhöhle oder der lufthaltigen Höhlen des Schädels entstehe, wurde in Betracht gezogen; es spricht dagegen: der normalerweise leere Schall, die große Verschiedenheit in der Intensität bei verschiedenen

Aus der Universitäts-Kinderklinik in Gießen.

\section{Ueber die Perkussion des Schädels bei Kindern und ihre diagnostische Verwertung. ${ }^{2}$ )}

\section{Von Prof. Dr. Hans Koeppe.}

Bei einem Fall von Hirntumor eines zweijährigen Knaben fand ich beim Beklopfen dès Schädels zur Feststellung zirkumskripter Stellen von Schmerzhaftigkeit einen hohen tympanitischen Schall und das Geräusch des gesprungenen Topfes - Schettern oder Scheppern. Dies war so auffallend und befremdend, da $B$ ich unwillkürlich $\mathrm{zu}$ der Vorstellung kam, innerhalb der Schädelhöhle müsse Luft sein, zumal auch noch der hohe tympanitische Schall stets am stärksten beim Belklopfen der höchstliegenden Schädelstelle gehört wurde und mit Lagewechsel des Kopfes sich die Stelle höchster Tympanie entsprechend verschob. Dies Verhalten konnte ich mit dem behandelnden Kollegen die ganze Zeit bis zum Tode des Kindes beobachten. Während dieser Zeit blieb die Tumorpapille beiderseits die wesentlichste Stütze der Diagnose, eine Lokalisation des Tumors war unmöglich, sodaß auch aus diesem Grunde dem Ergebnis der Obduktion mit Spannung entgegengesehen wurde. Es fand sich der Tumor, aber keine Luft im Schädel.

Später erfuhr ich, daß das Schettern ein dem Chirurgen bekanntes Symptom bei Hirntumor sei, doch die Tympanie des Schádels beim Beklopfen blieb mir so befremdend wie zuvor

Die Lehrbücher der Diagnostik ${ }^{3}$ ) bringen nichts über eine Perkussion des Schädels und lehren, daß nach der Er fahrung lauter Schall den lufthaltigen, leiser den luftleeren Organen, seien sie kompakt oder mit Flüssigkkeit gefüllt, zukommt, der tympanitische Schall auch durch die Spannung der Membran, die den Luftraum umschließt, beeinflußt wird. Schon unsere eine Beobachtung zeigt also, daß auch über dem kompakten und teilweise mit Flüssigkeit gefüllten Schädel beim Kinde der Schall nicht unbedingt leise, leer sein muB, sondern auch tympanitisch sein kann und

1) Sehr lehrreich ist nach dieser Richtung die von $\mathrm{Fahr}$ ausgeführte anatomis'he Lntersuchung eines Falles von rasch tödlich verlaufener Meningitis luica neun Wochen nach dem Primäraffekt (Derm. Wschr. $59 \mathrm{Nr} .38$ ). Vor allem sei auf W e chsel m a n n s grundlegende Monographie verwiesen: I 'er gegenwärtige Stand der Salvarsantherapie in Beziehung zur Pathogenese und Heilung der Syphilis (Berlin 1912). Zum Beleg des Satzes, ,daB schon in der präroseolen Periode. wenn nur der Primäraffekt besteht, das Gehirn unter Umständen schwer von Syphilis befallen sein kann", wird die gesamte Literatur herangezogen. Ein Fall aus der russischen Literatur betraf einen Offizier. der am Ende der dritten Woche nach dem Koitus nach Diagnose der Sklerose sich erschossen hatte. Die histologische Untersuchung ergab u. a. eine Periarteriitis auch der Endarterien der HirngefäBe und eine Verdickung winzigster Gefäße in der Pia.

2) Vortrag in der Medizinischen Gesellschaft in Gießen, 20. XI. 1918.

3) z. B. Le u b e, Diagnose der inneren Krankheiten 1895; S a h li Lehrb. der Untersuchungsmethoden 1913; B r u gs ch u. S chitten. h e 1 m, Lehrb. klin. Untersuchungsmethoden 1916 vielleicht auch die Beobachtung, daß ein Unterschied des Tones bei geöffnetem und dann bei geschlossenem Munde nicht eindeutig festzustellen war

Als weitere Schwierigkeit kommt noch hinzu, daß normalerweise beim Säugling Tympanie beim Beklopfen des Schädels zu hören ist und zuweilen auch Schettern, sodaB auch das Alter des Untersuchten in Betracht kommt. Die Untersuchungen am Säugling führten aber schließlich doch zu einem gewissen Abschluß und $\mathrm{zu}$ einer einheitlichen Auffassung der Erscheinung.

Am besten hört man die Tympanie, wenn der Säugling auf dem Rücken und der Hinterkopf aufliegt und man an den Scheitelbeinen perkutiert, also seitlich klopft, Beklopfen des Stirnbeins, also der höchst gelegenen Stelle, gibt meist leeren Schall. Zuweilen kann man auch leises Schettern hören und mit den aufgelegten Fingerspitzen der anderen Hand öfter noch leichte Vibration fühlen. Beklopfen der Fontanelle gibt gewöhnlich leeren Schall, manchmal auch Tympanie.

Bei gesunden Säuglingen ist der tympanitische Klang auch nicht immer deutlich $\mathrm{zu}$ erkennen, ist stets wenig intensiv und läßt sich verhältnismäßig schlecht demonstrieren. Hohe oder starke Tympanie, oft mit Schettern, findet sich bei Rachitis in Fällen, bei denen die große Fontanelle ziemlich gespannt ist. Anderseits wieder zeigte gerade ein Fall hochgradigster Rachitis mit enormer Kraniotabes, wobei der ganze Schädel wie eine mit Wasser gefüllte Blase dalag, vollkommen leisen, leeren Schall, gleichviel, wo man klopfte, keine Spur von Tympanie. Beim Schreien der Kinder wird die Tympanie stärker, man fühlt dann auch, wie die Fontanelle sich stärker spannt. Vollkommen leerer Schall fand sich bei den Fällen von Hydrozephaloid, bei denen dann mit zunehmender Genesung der leere Schall verschwand und allmählich Tympanie eintrat, gleichzeitig mit besserer Spannung der Fontanelle. Hochgradige Tympanie zeigte ein Fall von Spina bifida, derselbe Fall nach spontaner Ruptur des Sackes vollkommen leeren schall. Bei einem anderen Fall von Spina bifida konnte man beim Ablassen des Liquors durch Lumbalpunktion den Uebergang von hoher Tympanie zu vollkommen leerem Schall unmittelbar beobachten. Das Umgekehrte ließ sich bei demselben Fall von Spina bifida demonstrieren, wenn man durch Lumbalpunktion Liquor nur so weit abließ, bis der Schädelschall leer war; drückte man nun auf den nur noch halb gefüllten Sack, so spannte sich die Fontanelle. wieder, und tympanitischer Schall trat wieder auf

Da in der Spannung der großen Fontanelle der Druck des Innern des Schädels unmittelbar zum Ausdruck kommt, zeigen die Beobachtungen am Säugling, daß hier die Ursache des verschiedenen Schalls bei der Schädelperkussion in der Verschiedenheit des intrakraniellen Drucks und der dadurch bedingten Spannung der Schädelwandung zu suchen ist.

Verallgemeinern wir diese für den Säugling direkt beobachtete Anschauung auch für größere Kinder, so kommen wir zu der Hypothese: Schettern und tympanitischer Schädelschall sind ein Zeichen für einen erhöhten Druck innerhalb des Schädels, und die Intensität der Tympanie ist ein Maß für diesen Druck. 
Diese Hypothese erklärt zunächst zwanglos den Befund von Schettern und Tympanie bei verschiedenen Erkrankungen und zugleich den verschiedenen Grad der Intensität des Phänomens, den wir beobachteten, denn das Zeichen ist hiernach nicht als charalkteristisch für eine bestimmte Krankheit aufzufassen, sondern bezieht sich auf einen gewissen physikalischen Zustand des Untersuchten, der durch die verschiedensten physiologischen und pathologischen Bedingungen verursacht sein kann.

Natürlich genügt das nicht, sondern wir haben die Richtigkeit oder auch nur bedingte Gültigkeit der Hypothese noch zu prüfen, indem wir das neue Symptom für Hirndruck mit den bisher bekannten vergleichen und gleichzeitiges Auftreten nachweisen.

Für diese Prüfung kommen nur Tumorpapille, Sektionsbefund und direkte Messung des Liquordruckes gelegentlich der Lumbalpunktion in Betracht.

Nehmen wir von diesem Gesichtspunkt aus alle Fälle vor, bei denen wir Schettern oder Tympanie beim Beklopfen des Schädels fanden, so kämen zuerst die Fälle von Hirntumor, denn bei Hirntumoren erreicht die allgemeine Drucksteigerung im Schädelraum wohl den höchsten Grad. In der Tat zeigten von den beobachteten sechs Fälle $(2,3,7,10,11$ und 12 Jahre alt) mit der Diagnose Hirntumor den höchsten Grad von Tympanie und Schettern, und die Augenuntersuchung ergab zweimal Stauungspapille, zweimal postneuritische Atrophie nach Stauungspapille, zweimal Neuritis optica im Bilde der Staungspapille. Was die Lumbalpunktion anbelangt, so ergibt diese bei Hirntumoren keinen absoluten Aufschluß über den Hirndruck, da hier der Hirndruck im wesentlichen durch den soliden, wachsenden Tumor bedingt ist und der Druck, unter dem der Liquor steht, nicht gemessen werden kann, da der Liquor durch Verlegung des Foramen magnum oft nicht abfließt. Zweimal ist bei unseren sechs Fällen die Lumbalpunktion nicht gemacht worden, bei einem von diesen fand sich bei der Sektion der Hirntumor; bei drei Fällen flossen nur einige Tropfen Liquor $\mathrm{ab}$; auch von diesen wurde ein Fall seziert und ein Tumor gefunden. Beim letzten der sechs Fälle ergab die Punktion einen Druck von über $380 \mathrm{~mm}$ Wasser, also einen außerordentlich erhöhten Druck, aber nach Abfluß von Liquor und Sinken des Druckes blieb das Schettern bestehen, augenscheinlich, weil eben der durch den Tumor selbst bedingte hohe Druck noch nach $\mathrm{Ab}$ flu $\beta$ des Tumors weiterbestand.

Da bei Meningitis eine Lumpalpunktion an sich angezeigt ist, eignen sich diese Fälle ebenfalls zur Prüfung unserer Annahme, daß Tympanie des Schädels durch erhöhten intrakraniellen Druck bedingt sei. Ein Fall von Streptokokkenmeningitis zeigte Schettern, die Punktion ergab Eiter, ein zweiter Fall $130 \mathrm{~mm}$ Druck. Bei einem Fall von Meningokokkenmeningitis ist bei der Aufnahme Schettern notiert, bei der Punktion erhöhter Druck, nach Ablassen von Liquor ist das Schettern geringer, verschwindet in den nächsten Tagen, und wiederholte Punktionen ergeben keinen erhöhten Druck mehr. Der Fall ging in Heilung über.

Von tuberkulöser Meningitis kamen 15 Fälle zur Beobachtung, alle mit Schädeltympanie, teilweise mit Schettern. Bei fünf Fällen mit Obduktionsbefund wurden acht Punktionen gemacht, fünfmal war der Druck erhöht, dreimal nicht. Dreimal wurde Aenderung des Schädelschalles (Abschwächung der Tympanie) nach Ablassen von Liquor beobachtet, einmal nicht. Viermal ist nichts notiert. Bei den zehn anderen Fällen ohne Obduktionen wurden elf Punktionen gemacht, bei denen neunmal der Druck erhöht gefunden wurde, viermal Abschwächung der Tympanie nach Ausfließen des Liquors, dreimal keine, dreimal ist nichts notiert.

In den meisten Fällen ( 16 mal bei 22 Punktionen) also konnte bei Schädeltympanie durch die Lumbalpunktion erhöhter Druck nachgewiesen werden, wodurch die Gültigkeit unserer Hypothese, daß Tympanie des Schädels bei-Kindern durch erhöhten intrakraniellen Druck hervorgerufen wird, eine kräftige Stütze erhält

Diese Zahlen gewinnen noch an überzeugender Kraft, wenn man bedenkt, daß ja bei der Lumbalpunktion nicht immer der wirkliche Gehirndruck gemessen wird und daß der Hirndruck groß sein kann, auch wenn der Liquordruck nicht erhöht ist, wie bei Meningealödem, sulziger Durchtränkung der Hirnhaut, Tuberkeln usw. Also ein bei der Lumbalpunktion gefundener, nicht erhöhter Druck entkräftet die Annahme nicht, daß auch in solchen Fällen die Schädeltympanie erhöhten Gehirndruck anzeigt.

Weit überzeugender ist aber die unmittelbare Beobachtung, besonders das Ablklingen und Schwăcherwerden bis zum Verschwinden der Tympanie beim Ablassen von Liquor.

Ich halte daher die aufgestellte Hypothese der Wahrheit mindestens sehr. nahekommend und bin geneigt, nunmehr jede einwandfrei beobachtete Tympanie beim Beklopfen des kindlichen Schädels als Zeichen erhöhten Gehirndrucks anzusehen, glaube auch, daß durch dieses Symptom erhöhter Hirndruck nunmehr bäufiger und bei verschiedenen Krankheitszuständen erkannt wird, unter Umständen für diese eine Erklärung abgeben kann.
Bis jetzt fand ich tympanitischen Schädelschall bei Miliartuberkulose, bei dem klinischen Bilde des Meningismus, vielfach bei Otitis, vereinzelt bei hohem Fieber; bei Pneumonie, bei Grippe.

Mit der Aufstellung der Schädeltympanie als allgemeines Symptom für erhöhten Hirndruck und dessen Nachweis bei einer ganzen Reihe verschiedenartiger Krankheiten erschien mir nun nach und nach dieses Symptom für die Diagnostik immer weniger wertvoll zu werden. Dies gilt aber nur, wenn man auf Grund dieses ein en Symptoms eine Diagnose stellen wollte; im Verein mit anderen Symptomen ist es sehr wertvoll und leistet viel.

Ich habe schon erwähnt, daß im Anfang meiner Untersuchungen in drei Fällen erst die Schädeltympanie den Anlaß gab, den Augenhintergrund zu untersuchen, wonach die Diagnose Hirntumor aus der Tumorpapille gestellt wurde. In einem Falle, der vorher als chronische Nephritis angesehen wurde, machte das Symptom die Diagnose Hirntumor wahrscheinlich. In einem Fall zerebraler Kinderlähmung bei einem zweijährigen Kinde veranlaßte die Schädeltympanie ebenfalls Untersuchung des Augenhintergrundes, durch welche Sehnervenatrophie festgestellt wurde, aber da die Fontanelle noch nicht geschlossen war, glaube ich das Schädelsymptom hierauf zurückführen zu müssen, bei der Sektion fand sich aber doch eine Geschwulst, ein Hygrom der Dura mater. Hier hätte also das Symptom auf die richtige Diagnose hinlenken können, wie es in einem anderen Falle zutraf. Der Klinik wurde ein Fall (zweieinhalb Jahr alt) schwerer Blutfleckenkrankheit geschickt, typische Purpura haemorrhagica im Endstadium, mit schwerer Benommenheit. Als Ursache wurde an eine Sepsis gedacht. Die Schädeltympanie mit Anklang an Schettern veranlaßte die Lumbalpunktion, bei der unter erhöhtem Druck sich trüber Liquor entleerte, in dem durch bakteriologische Untersuchung Meningokokken nachgewiesen wurden.

Im Gegensatz hierzu brachte die Schädeltympanie in zwei Fällen auf die falsche Diagnose Meningitis, während in Wirklichkeit nur erhöhter Gehirndruck vorlag. Bei dem einen Fall $H$. war der Schädelschall bei der Aufnahme leer, nach einigen Tagen war deutliche Schädeltympanie zu finden, bei der Lumbalpunktion wurde $300 \mathrm{~mm}$ Wasserdruck gemessen, die Tympanie ging zurück und blieb undeutlich, deshalb keine Wiederholung der Punktion. Die Diagnose Meningitis tuberculosa mußte bei der Selition in Peritonitis tuberculosa geändert werden. Der andere Fall L. (zwei Jahre drei Monate) wurde vom Kollegen eingeschickt mit der Diagnose: Ruhr oder Meningitis. Der Schädelschall war ausgesprochen tympanitisch. Bei der Lumbalpunktion flossen nur einige Tropfen Liquor ab. Die Diagnose wurde auf Meningitis und Dickdarmkatarrh gestellt, bei der Autopsie fand sich außer starker akuter Entzündung des Dickdarms Meningealödem und Hyperämie des Gehirns und seiner Häute, wodurch der tympanitische Schädelschall bei fehlendem Liquordruck sich glatt erklärt.

In zwei anderen Fällen, von denen der eine (S.) als Darmkatarrh in die Klinik geschickt wurde, der andere (Sch.) wegen Spina ventosa aufgenommen wurde, konnte als erstes und zunächst einziges Zeichen der sich einstellenden tuberkulösen Meningitis die Schädeltympanie festgestellt werden

Noch ziemlich ungeklärt, aber sehr beachtenswert erscheinen die Fälle mit Schädeltympanie bei Otitis. Ich müßte bei diesen auf die einzelnen Krankengeschichten in extenso eingehen, was jetzt zu weit führen würde, und verweise auf die ausführliche Veröffentlichung, welche auch die Krankengeschichten der anderen besprochenen Fälle bringen wird. Es besteht jedoch für mich kein Zweifel, daß gerade diese Fälle für die Deutung der Schädeltympanie und die Schätzung ihres diagnostischen Wertes oft recht große Schwierigkeiten bereiten werden und noch viele Beobachtungen nötig machen. Zu bedenken ist noch, daß, wie bei der Perkussion anderer Organe, auch bei der Schädelperkussion eben dem subjektiven Empfinden ein ziemlicher Spielraum gelassen ist. Trotz alledem möchte ich schon jetzt meine Beobachtungen bekanntgeben und zur Nachprüfung derselben auffordern, da ich überzeugt bin, daß sich die Perkussion des Schädels als Untersuchungsmethode weiter ausbauen und an diagnostischem Werte gewinnen wird. Von großem Interesse wird es sein, zu erfahren, wie sich diese Verhältnisse bei Erwachsenen erweisen. 